PARASITE BIOLOGY

\title{
Cell division goes back to its roots
}

During cell division of the intracellular apicomplexan parasite Toxoplasma gondii, the formation of emergent daughter buds is dependent on the apical microtubules and the inner-membrane complex (IMC). However, the mechanism by which cell division is coordinated with mitosis and organelle segregation is

emerging daughter bud and, by doing so, ensures correct segregation of the nucleus and other organelles.

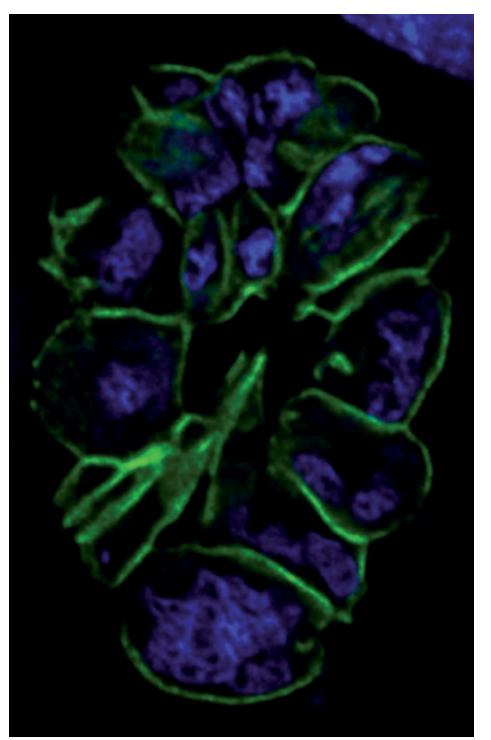

Immunofluorescence image showing that depletion of striated fibre assemblin (SFA3) causes a loss of coordination between nuclear division and budding, resulting in the accumulation of multiple nuclei within a single cell (blue). Inner-membrane complex 1 (IMC1) is shown in green. Image courtesy of B. Striepen and M. Francia, University of Georgia, Athens, USA. not well understood. Writing in PLoS Biology, Francia et al. now show that homologues of algal flagellum components act as spatial and temporal organizers of T. gondii cell division.

In T. gondii, chromosomes are constantly tethered to the centrosomes (the main microtubuleorganizing centre of the parasite cell), as are the apicoplast and Golgi. Previous work had identified T. gondii homologues of striated fibre assemblin (SFA), a component of the flagellar basal body in green algae, and had shown these homologues to localize to the vicinity of the centrosome. To investigate whether these proteins have a role in cell division, the authors tagged SFA2 and SFA3 and monitored their localization by immunofluorescence microscopy. The proteins were not expressed in interphase cells prior to cell division, but shortly after centrosome duplication the proteins colocalized in short fibre-like structures, with one end of the structure close to or overlapping the centrosome, and the other end containing a spiralling hook shape and extending towards the apical end of the daughter bud. Growth in fibroblasts was severely impaired when parasites were depleted for either SFA2 or SFA3, and cells lacking the SFA fibre were excessively large and contained multiple nuclei, suggesting that the defect was not in mitosis but in the subsequent budding of daughter cells. In agreement with this idea, when antibodies against IMC components were used to label the pellicle of the emerging daughter bud, cells depleted of SFA2 or SFA3 showed either aberrant or no daughter cell buds. Finally, using time-lapse immunofluorescence imaging and transmission electron microscopy, the authors visualized the SFA fibre elongating away from the centrosome and forming a physical connection with the tip of the daughter bud.

The authors suggest that the SFA fibre tethers the centrosome to the apical tip of each emerging daughter bud and, by doing so, ensures correct segregation of the nucleus and other organelles. The apicomplexa are thought to have evolved from a photosynthetic aquatic alga, losing flagella along the way; these findings indicate that they might have retained some components of the flagellar machinery for use during division.

Andrew Jermy

ORIGINAL RESEARCH PAPER Francia, M. E. et al. Cell division in apicomplexan parasites is organized by a homolog of the striated rootlet fiber of algal flagella. PLoS Biol. 10, e1001444 (2012) 